\title{
Integrated Machian model of a light speed rotating universe
}

\author{
U.V.S. Seshavatharam ${ }^{1}$ and S. Lakshminarayana ${ }^{2}$ \\ ${ }^{1}$ Honorary faculty, I-SERVE, Survey no-42, Hitech city, Hyderabad-84,Telangana, India \\ 2 Dept. of Nuclear Physics, Andhra University, Visakhapatnam-03,AP, India \\ Emails: seshavatharam.uvs@gmail.com (and) lnsrirama@gmail.com \\ Orcid numbers : 0000-0002-1695-6037 (and) 0000-0002-8923-772X
}

\begin{abstract}
Unification point of view, quantum cosmology must be given a priority and one should make a note that, Spin is a basic property of quantum physics and rotation is a very common experience. In this context, we have developed a quantum model of cosmology associated with Machian universe having Planck scale origin, total dark matter, light speed rotation and equality of gravitational self energy density and thermal energy density. We would like to appeal that, observed cosmic radius and age seem to be shortened by 146.3 times their actual magnitudes.
\end{abstract}

Keywords: Mach's Universe; Planck scale; Cosmic Angular velocity; Galactic applications; shortened radius; shortened age;

\section{Introduction}

Even though standard model of cosmology is standing on 5 pillars namely, big bang, inflation, super luminal expansion, dark matter and dark energy, we would like to emphasize that,

1) James Peebles, the famous cosmologist and 2019 Nobel laureate, strongly believes that Big bang is an inappropriate concept in understanding the universe. Readers are encouraged to visit, https://phys.org/news/2019-11-cosmologist-lonely-big-theory.html

2) Theoretically big bang, inflation, dark energy and super luminal expansion are no way connected with Planck scale which is having a major role in understanding quantum cosmology having information passing at speed of light.

3) Experimentally so far no one could understand big bang, inflation, dark energy, dark matter and super luminal expansion with reference to any underground or ground or satellite based experiment.

4) Big bang, inflation and Super luminal expansion are no way giving a clue for unifying general theory of relativity and quantum mechanics.

5) Even though most of the cosmological observations are being studied and understood with photons that propagate with speed of light, it is very unfortunate to say that, most of the cosmologists are strongly believing in hypothetical 'super luminal expansion' of space. Recently detected gravitational waves that are supposed to originate from massive black holes are also confirmed to be moving at speed of light. If so, superluminal expansion can be considered as a pure human intellectual concept having no experimental support.

6) Big bang, Inflation and Dark energy are inference based intellectual concepts having no proper physical base and probably may misguide the future generation.

7) Compared to Big bang, inflation, dark energy and superluminal expansion, dark matter is having some sort of physical support in terms of an unknown, unidentified and unseen elementary particle having an heuristic gravitational attractive property. In addition to that, ultimately somehow, one should suppose the existence of some kind matter for understanding the unexpected massive nature of trillions of observed galaxies.

8) According to Kansas State University's very recent study conducted by Shamir L [1], an analysis of more than 200,000 spiral galaxies has revealed unexpected links between spin directions of galaxies and the structure formed by these links might suggest that the early universe could have been spinning. For a brief report readers are encouraged to visit: https://www.k-state.edu/media/newsreleases/202006/study-suggests-universe-has-defined-structure.html.

9) It is better to understand and develop models of cosmology based on well supported physical concepts rather than extraordinary physical hypothesis that demand super-normal efforts.

In this short report, with reference to life cycle of living creatures, un-quantified pre and post physical conditions of 'big bang' [2], unidentified reasons pertaining to presumed exponential expansion in cosmic size associated with inflation [3,4], unaccounted exponential increase in cosmic time associated with presumed cosmic inflation and independent of redshift data, we have taken an initiative in developing a quantum model of 
cosmology associated with Machian universe having Planck scale origin, total dark matter, light speed expansion, light speed rotation, equality of gravitational self energy density and thermal energy density, radially decreasing internal acceleration and radially increasing anisotropy. We have elaborately explained our views in recently published papers $[5,6,7]$. Readers are encouraged to go through.

\section{Motivating points}

We appeal that,

1) Einstein, the founder of general theory of relativity, strongly believed and greatly inspired by Ernst Mach's cosmological concept of 'influence of cosmic distance back ground'. The extent to which general relativity is actually Machian is, however, the subject of great controversy [8].

2) As known physics is helpless in quantifying 'Big bang' physical conditions, we propose a mechanism of 'growing Planck ball'. This logic seems to be simple and easy to implement.

3) As there is no proper theoretical reasoning for the origin of 'inflation', it's well believed end results like 'flatness', 'homogeneity' and 'isotropy' can also be understood with continuous and prolonged light speed expansion. This logic is natural, non-controversial, simple to follow and simple to implement.

4) As an end result of inflation, standard cosmology is giving a top priority for exponential cosmic expansion in size, but no way considering its corresponding exponential increase in cosmic time. When it is strongly believed that, space-time are inseparable quantities, it seems illogical to believe in the standard model of narrow time line of $\left(1 / H_{0}\right)$ associated with post inflation.

5) We stress the point that - without a radial in-flow of matter in all directions towards one specific point, one cannot expect a big crunch and without a big crunch, one cannot expect a big bang. Really if there was a 'big bang' in the past, with reference to formation of big bang as predicted by GTR and with reference to the cosmic rate of expansion that might have taken place simultaneously in all directions at a 'naturally selected rate' about the point of big bang - 'point' of big bang can be considered as the characteristic reference point of cosmic expansion in all directions. Thinking in this way, to some extent, point of big bang can be considered as a possible centre of cosmic evolution. If so, thinking about a centre less universe is illogical.

6) 'Space' and 'matter' are inseparable cosmic entities and like matter, space cannot travel faster than speed of light.

\section{Assumptions}

We appeal that, understanding cosmology with two or three assumptions seems to be insufficient. In this context, we have developed 9 assumptions under four groups. First group constitutes 3 assumptions associated with cosmic geometry, thermal behavior, angular velocity and expansion rate. Second group constitutes two assumptions associated with galactic dark matter, visible matter, flat rotation speeds and radii. Third group constitutes two assumptions associated with galactic red shifts and distances. Fourth group constitutes two assumptions associated with cosmic radius, age and expansion speed. With these set of assumptions it is possible to understand the observed universe in all aspects. With further study, assumptions can be refined and minimized in a better way.

\section{Group-1:}

Assumption-1: By following Mach's principle [8,9,10], right from the beginning of Planck scale, universe is rotating with speed of light from and about the cosmic centre. If, $G M_{t} \cong c^{2} R_{t}, \quad R_{t} \cong G M_{t} / c^{2} \cong c / \omega_{t}$, $M_{t} \cong c^{3} / G \omega_{t}$, where $M_{t}=$ cosmic mass, $R_{t}=$ cosmic radius and $\omega_{t}=$ cosmic angular velocity. 
Assumption-2: Right from the beginning of Planck scale, at any stage of cosmic expansion, gravitational self energy density and thermal energy density are equal in magnitude [7,11,12]. Its results are, 1) $-\frac{3}{5} \frac{G M_{t}^{2}}{R_{t}} \div \frac{4 \pi}{3} R_{t}^{3} \cong-\frac{9 G M_{t}^{2}}{20 \pi R_{t}^{4}} \cong-\frac{9 \omega_{t}^{2} c^{2}}{20 \pi G}$, 2) $-\frac{9 G M_{t}^{2}}{20 \pi R_{t}^{4}}+a T_{t}^{4} \cong 0$ and $\frac{9 \omega_{t}^{2} c^{2}}{20 \pi G} \cong a T_{t}^{4}$.

Assumption-3: At any stage of cosmic evolution, ratio of Hubble parameter to angular velocity can be expressed as, $H_{t} / \omega_{t} \cong\left[1+\ln \left(\omega_{p l} / \omega_{t}\right)\right] \cong\left[1+\ln \left(T_{p l} / T_{t}\right)^{2}\right] \cong \Upsilon_{t}$ where $\left(\omega_{p l}, T_{p l}\right)=$ Planck scale angular velocity and temperature respectively, $T_{t}=$ cosmic temperature at time $t$ and $\gamma_{t}=$ Ratio of $H_{t}$ and $\omega_{t}$.

\section{Group-2:}

Assumption-4: Galactic dark matter $\left(M_{G d}\right)_{t}$ and visible matter $\left(M_{G v}\right)_{t}$ are interrelated in such way that, $M_{G t} \cong\left(M_{G d}\right)_{t}+\left(M_{G v}\right)_{t}$ and $\frac{\left(M_{G d}\right)_{t}}{\left(M_{G v}\right)_{t}} \cong \sqrt{\frac{\left(M_{G v}\right)_{t}}{M_{X t}}}$ where $M_{X t} \cong\left(M_{t}^{3} M_{p l}\right)^{\frac{1}{4}} /\left(1+\sqrt{\gamma_{t}}\right)=$ time dependent darkvisible reference mass unit.

Assumption-5: Galactic flat rotation speed can be expressed as, $\frac{V_{G t}}{c} \cong\left(\frac{M_{G t}}{M_{t}}\right)^{\frac{1}{4}}$ where $M_{t}=\operatorname{cosmic}$ total mass.

\section{Group-3:}

Assumption-6: At present, if $\lambda_{O}$ is the wave length of light received from observed galaxy and $\lambda_{L}$ is the wave length of light in laboratory, actual galactic distances can be expressed as,

$$
\begin{aligned}
d_{G a} \cong z_{\text {new }}\left(\frac{\gamma_{0}}{\gamma_{z}}\right)\left(\frac{c}{\omega_{0}}\right) \cong\left(\frac{z}{z+1}\right)\left(\frac{\gamma_{0}}{\gamma_{z}}\right)\left(\frac{c}{\omega_{0}}\right) & \left.\begin{array}{l}
z_{\text {new }} \cong\left(\frac{\lambda_{O}-\lambda_{L}}{\lambda_{O}}\right) \cong\left[1-\left(\frac{\lambda_{L}}{\lambda_{O}}\right)\right] \leq 1 \\
\text { where }\left\{\begin{array}{l}
\text { and } z \cong\left(\frac{\lambda_{O}-\lambda_{L}}{\lambda_{L}}\right) \cong\left(\frac{\lambda_{O}}{\lambda_{L}}-1\right) \\
\gamma_{z} \cong 1+\ln \left(\frac{\omega_{\text {pl }}}{\omega_{z}}\right)
\end{array}\right\} \text { where } \omega_{z} \cong \frac{\omega_{0}}{z_{\text {new }}}
\end{array}\right\}
\end{aligned}
$$

Assumption-7: At present, shortened galactic distances can be expressed as,

$$
d_{G s} \cong \frac{d_{G a}}{\gamma_{0}} \cong z_{\text {new }}\left(\frac{c}{\gamma_{z} \omega_{0}}\right) \cong\left(\frac{z}{z+1}\right)\left(\frac{c}{\gamma_{z} \omega_{0}}\right)
$$

\section{Group-4:}

Assumption-8: Cosmic radius can also be expressed as, $R_{t} \cong \sqrt{(c t)\left(\frac{c}{\omega_{t}}\right)}$. Then, $t \times \omega_{t} \cong 1$. Hence, $t \cong \frac{1}{\omega_{t}}$.

Assumption-9: At any stage of cosmic evolution, shortened cosmic age can be expressed as, $t_{s} \cong \frac{t}{\gamma_{t}} \cong \frac{1}{\gamma_{t} \omega_{t}} \cong \frac{1}{H_{t}}$. 
Note-1: Based on light speed expansion models [7], if one is willing to consider $R_{t} \cong c t$, then based on assumption- $6,(c t) \cong\left(\frac{c}{\omega_{t}}\right)$.

Note-2: Based on assumptions (2) and (8), it is possible to show that, $T_{t} \cong\left(\frac{9 c^{2}}{20 \pi G a t^{2}}\right)^{\frac{1}{4}} \cong\left(\frac{2.247 \times 10^{10}}{\sqrt{t}}\right) \mathrm{K}$. This can be compared with currently believed Time-Temperature relation associated with radiation dominated era.

\section{Understanding galactic shortened distances and their corresponding comoving distances}

Based on assumption-7, estimated galactic distances are approximately equal to the distances estimated with standard cosmology where matter density fraction is 0.32 and dark energy density fraction is 0.68 . For example, for a redshift of $\mathrm{z}=1$, estimated and standard model distances are 7.4 Gly and 8.07 Gly respectively. For a redshift of $z=11$, estimated and standard model distances are 13.54 Gly and 13.56 Gly respectively. Similarly for $z=1100$, estimated and standard model distances are 14.74 Gly and 13.98 Gly respectively. In addition to that, currently believed comoving distances can be approximated with, $\left(d_{G s}\right)_{c o m} \cong \exp \left(\frac{z}{z+1}\right)\left(d_{G s}\right) \cong \exp \left(\frac{z}{z+1}\right) \times\left(\frac{z}{z+1}\right) \times\left(\frac{c}{\gamma_{z} \omega_{0}}\right)$.

\section{Understanding cosmic rotation}

According to Vladimir A. Korotky, Eduard Masár and Yuri N. Obukhov [13]: "In observational cosmology, the main difficulty for detecting a global rotation is its smallness-less than $10^{-13} \mathrm{rad} /$ year according to the generally accepted assessment. It is impossible in the Universe to distinguish the direction corresponding to the axis of rotation, with respect to which one could notice deviations (in the standard tests) from the Friedman standard cosmology. In theoretical cosmology, the main difficulties are related, on the one hand, to the lack of simple models of an expanding and rotating Universe in general relativity (GR) similar to FriedmanRobertson-Walker models. On the other hand, there are no convincing predictive effects of cosmic rotation that are consistent with the capabilities of the equipment of modern astronomical observatories".

In this context, we strongly propose that, as 'spin' is a basic property of quantum mechanics, from the subject point of quantum gravity, universe must have 'rotation'. If it is assumed that, universe is a Machian sphere, it is quite natural to expect 'cosmic rotation'. Considering the 6 major applications of dark matter in view, by estimating the currently believed dark mass of a galaxy with its corresponding visible mass and by replacing the 'acceleration' parameter $\left[\approx\left(\mathrm{cH}_{0}\right) / 6\right]$ of Modified Newtonian Dynamics (MOND) $[14,15]$ with current cosmic angular acceleration $\left(c \omega_{0}\right)$, we try to fit the flat rotation speed of a galaxy. One most interesting as well as speculative point is that, even though MOND approach is 'the best' in fitting galactic rotation curves, its back ground physics is unclear with respect to galactic structures and cosmic acceleration parameter $\left(c H_{0}\right)$. It can be confirmed with the conclusion section of recent paper authored by Stacy McGaugh [16]. Here, our approach is having 5 different actions:

1) To estimate the generally believed dark mass of a galaxy.

2) To implement cosmic angular acceleration in understanding galactic structure.

3) To fit flat rotation speed of a galaxy.

4) To estimate the radius of a galaxy.

5) Finally, to reveal the existence of cosmic rotation.

\section{Basic applications of cosmic angular velocity}

Simplified cosmic temperature and angular velocity relation can be expressed as, $k_{B} T_{t} \cong 0.6831 \hbar \sqrt{\omega_{t} \omega_{p l}}$ where $\omega_{p l} \cong \sqrt{c^{5} / G \hbar}=$ Planck scale angular velocity. Interesting point is that, $\hbar \sqrt{\omega_{t} \omega_{p l}}$ can be considered as a 
representation of rest energy of cosmic graviton [17,18]. Matter creation rate can be expressed as, $M_{t} \omega_{t} \cong\left(c^{3} / G\right)$ and $M_{t} \cong c^{3} / G \omega_{t} \cong c^{3} t / G$.

With reference to currently believed cosmic temperature of $T_{0} \cong 2.722 \mathrm{~K}$ [19] and by considering $T_{p l} \cong \sqrt[4]{9 \omega_{p l}^{2} c^{2} / 20 \pi G a} \cong 9.67791 \times 10^{31} \mathrm{~K}$, an attempt is made to define the current cosmic angular velocity $\omega_{0}$ and Hubble parameter $H_{0}$. Estimated magnitudes are, $\omega_{0} \cong 1.4674 \times 10^{-20} \mathrm{rad} . \mathrm{sec}^{-1}$ and $H_{0} \cong 66.24 \mathrm{~km} / \mathrm{Mpc} / \mathrm{sec}$ respectively. Corresponding current cosmic radius and mass are $R_{0} \cong c / \omega_{0} \cong 2.043 \times 10^{28} \mathrm{~m}$ and $M_{0} \cong c^{3} / G \omega_{0} \cong 2.751 \times 10^{55} \mathrm{~kg}$ respectively.

\section{To estimate the magnitude of galactic dark matter}

Galactic dark matter seems to have a major role in understanding 6 different issues pertaining to many massive galaxies. They are:

1) Galactic formation and evolution.

2) Galactic rotational curves.

3) Gravitational lensing.

4) Galactic collisions.

5) Motion of galaxies within galaxy clusters.

6) Cosmic microwave background fluctuations.

In this context, we would like to suggest the following two points.

1) By giving a priority to galactic 'visible matter', dark matter can be expressed as, Dark matter $\propto$ (Visible matter $)^{\frac{3}{2}}$.

2) By giving a priority to galactic 'dark matter', visible matter can be expressed as, Visible matter $\propto(\text { Dark matter })^{\frac{2}{3}}$.

By considering current cosmic mass as 'complete dark matter' and considering visible matter as a cosmological action of transformation of dark matter, an attempt is made to estimate galactic dark matter associated with galactic visible mass via a characteristic reference mass unit [5,6,7] of $M_{X} \cong \sqrt[4]{M_{0}^{3} M_{p l}} /\left(1+\sqrt{\gamma_{0}}\right) \cong 3.5 \times 10^{38} \mathrm{~kg}$ where $M_{p l} \cong \sqrt{\hbar c / G}$ and $\gamma_{0} \cong 146.3$. It needs further study.

\section{Galactic applications of cosmic angular velocity}

By considering the sum of galactic dark matter and visible matter along with current maximum cosmic angular acceleration $c \omega_{0}$, an attempt is made to fit galactic flat rotation speeds, radii, angular velocities and geometric mass densities. Corresponding relations can be expressed as follows.

$$
\left.\begin{array}{rl}
V_{G} & \cong \sqrt[4]{G M_{G}\left(c \omega_{0}\right)} \\
r_{G} \cong \sqrt{\frac{G M_{G}}{c \omega_{0}}} \\
\omega_{G} \cong \frac{V_{G}^{3}}{G M_{G}} \\
\rho_{G v} \approx\left[\frac{\left(V_{G}\right)}{c}\right] \sqrt{\frac{M_{G}}{M_{G v}}}\left(\frac{3 M_{G v}}{4 \pi r_{G}^{3}}\right) \\
\approx(1.0 \text { to } 30.0) \times 10^{-28} \mathrm{~kg} \cdot \mathrm{m}^{-3}
\end{array}\right\}
$$

where, $M_{G v}=$ Visible mass of galaxy 


$$
\begin{aligned}
& M_{G d}=\text { Dark mass of galaxy }=\sqrt[3]{M_{G v}} / \sqrt{M_{r e f}} \\
& M_{G}=M_{G v}+M_{G d} \\
& r_{G}=\text { Radius of galaxy } \\
& \omega_{G}=\text { Angular velocity of galaxy } \\
& \rho_{G v}=\text { Visible mass density of galaxy }
\end{aligned}
$$

Proceeding further, considering current cosmic radius as a scaling length and by considering average flat rotation speed of any two neighboring galaxies, their separation distance (490 Mly as expected by Baryonic Acoustic Oscillations) $[20,21]$ can be fitted with a relation of the form $\left[\sqrt{c V_{G}} / H_{0}\right]$ where $V_{G}$ represents flat rotation speed of any galaxy.

\section{Time line of nucleosynthesis}

According to standard model of cosmology, primordial nucleosynthesis seems to be happened in between first (3 to 20) minutes of big bang [22]. Expected approximate cosmic temperature range for this characteristic time period is $\left(10^{9}\right.$ to $\left.10^{7}\right) \mathrm{K}$. Based on our assumptions, cosmic Time-Temperature relation can be expressed as, $T_{t} \cong\left(9 c^{2} / 20 \pi G a t^{2}\right)^{\frac{1}{4}} \cong 2.247 \times 10^{10} / \sqrt{t}$ where, $t=$ Actual time expressed in seconds and $T_{t}=$ Cosmic temperature corresponding to actual time $t$. Here, we would like to emphasize that, this relation is very similar to the main stream consideration and very interesting point to be noted is that, this relation is absolutely free from Big bang concept. Only thing to be understood is - there exists two time scales - One is 'actual' time scale and the other one is 'shortened' time scale. For the same temperatures, shortened time scale can be represented by, $t_{s} \cong \frac{t}{\gamma_{t}} \cong \frac{1}{\gamma_{t}} \sqrt{\frac{9 c^{2}}{20 \pi G a T_{t}^{4}}}$.

\section{Issues connected with cosmological constant and horizon problem}

Considering the repulsive nature of Lambda term i.e. $\Lambda_{t}\left(\frac{c^{4}}{8 \pi G}\right)$, attractive nature of gravitational self energy density i.e. $-\left(\frac{9 G M_{t}^{2}}{20 \pi R_{t}^{4}}\right)$ and based on the results of assumption-3, if one is willing to consider Lambda term as $-\frac{9 G M_{t}^{2}}{20 \pi R_{t}^{4}}+\Lambda_{t}\left(\frac{c^{4}}{8 \pi G}\right) \cong 0$, it is possible to show that, $\Lambda_{t} \cong\left(\frac{9 G M_{t}^{2}}{20 \pi R_{t}^{4}}\right)\left(\frac{c^{4}}{8 \pi G}\right)^{-1} \cong 3.6\left(\frac{\omega_{t}^{2}}{c^{2}}\right)$. Here we would like to appeal that, compared to relation, $\Lambda_{t} \cong 3.6\left(\frac{\omega_{t}^{2}}{c^{2}}\right), \quad \Lambda_{t} \cong\left(\frac{9 G M_{t}^{2}}{20 \pi R_{t}^{4}}\right)\left(\frac{c^{4}}{8 \pi G}\right)^{-1}$ is having more physical meaning where $\left(\frac{c^{4}}{8 \pi G}\right)$ can be considered as a representation of constant gravitational binding force associated with cosmic evolution. Clearly speaking, attractive force being a constant, Lambda term decreases with decreasing gravitational self energy density. With reference to Planck scale, binding force being a cosmic constant, $\frac{\Lambda_{p l}}{\Lambda_{t}} \cong\left(\frac{M_{p l}^{2}}{M_{t}^{2}}\right)\left(\frac{R_{t}^{4}}{R_{p l}^{4}}\right)$. In this way, it seems possible to understand the issues connected with currently believed cosmological constant problem [23].

\section{Issues connected with cosmic expansion velocity and flatness problem}

We would like to emphasize the point that, galactic red shift data and its advanced analyzing patterns clearly show a serious controversy in understanding the true nature of cosmic rate of expansion. References [24] and [25] indicate an accelerating expansion. Reference [26] indicates a constant rate of expansion and reference [27] 
indicates a static universe. Most worrying thing is that, all these four references got published in main stream journals having very high impact. In this context, independent of the controversial galactic redshift data,

1) It is absolutely possible to consider cosmic temperature as a probe of understanding the true cosmic expansion rate.

2) By considering the actual rate of decrease in current cosmic temperature, future cosmic expansion rate can be understood.

At present, root mean square error in current cosmic microwave back ground radiation (CMBR) temperature is 18 micro Kelvin and average error is around 60 to 70 micro Kelvin. With further study and observations, it is necessary to continuously monitor the drop in CMBR temperature. Drop in CMBR temperature can be considered as a standard index for understanding the actual cosmic rate of expansion.

If one is willing to consider current expansion velocity as, $\mathrm{v}_{0} \cong \frac{R_{0}}{t_{0}} \cong\left(\frac{c}{\omega_{0}}\right) \div\left(\frac{1}{\omega_{0}}\right) \cong c$. Thus, at present, by considering light speed expansion and light speed rotation, there exists no scope for 'flatness' problem. It may also be noted that, according to Phillip Helbig [28, 29] - "flatness problem does not exist, not only for the cosmological models corresponding to the currently popular values of $\lambda_{0}$ and $\Omega_{0}$ but indeed for all FriedmannLemaitre models". In this context, only thing to be understood is - What is the intrinsic connection between universe, photons and light speed? Anyhow, independent of the galactic redshift data and its controversial analysis and by continuously measuring the rate of decrease in current cosmic temperature, future cosmic expansion rate can be understood confidently. As universe is assumed to be a model of light speed rotating object, there is a possibility cosmic anisotropy [30]. We would like to emphasize the fact that, the basic principles of cosmology were developed when the subject of cosmology was in its budding stage. Friedmann made two simple assumptions about the universe [31]. They can be stated in the following way.

1) When viewed at large enough scales, universe appears the same in every direction.

2) When viewed at large enough scales, universe appears the same from every location.

In this context, Hawking expressed that [32]: “There is no scientific evidence for the Friedmann's second assumption. We believe it only on the grounds of modesty: it would be most remarkable if the universe looked the same in every direction around us, but not around other points in the universe".

\section{Issues connected with cosmic radius and age}

Observable cosmic radius is just 3.2 times the Hubble radius and corresponding cosmic age is $\left(1 / H_{0}\right)$. Our model result of cosmic radius is 146.3 times the Hubble radius and corresponding light speed cosmic age is 146.3 times $\left(1 / H_{0}\right)$. We would like to emphasize that, observed cosmic radius and age are shortened forms and their actual magnitudes are 146.3 times higher. It needs further study.

\section{Issues connected with nature of dark matter}

We would like to appeal that, when total or most of the cosmic matter is believed to be in the form of dark nature having interactions only with gravitation, it may not be logical to attribute its nature to any known or unknown elementary particle supposed to be originating from interactions involved with elementary mass spectrum having negligible gravity at atomic, nuclear and electroweak scales.

\section{Issues connected with magnitude of galactic dark matter}

It may be noted that, with reference to recommended visible mass of Milk Way and its estimated dark mass, estimated flat rotation speed is $200 \mathrm{~km} / \mathrm{sec}$ and effective radius is $294 \mathrm{kpc}$ [33]. Alternatively, for any galaxy, by knowing the flat rotation speed, its total mass can be estimated with, $M_{G} \cong V_{G}^{4} / G c \omega_{0}$. By considering the relation, $M_{G} \cong M_{G v}\left(1+\sqrt{M_{G v} / M_{X}}\right)$ and with a simple computer program, visible and dark masses can be estimated. Proceeding further, galaxies whose visible mass approaches our reference mass unit of 
$M_{r e f} \cong 3.5 \times 10^{38} \mathrm{~kg}$, seems to possess very little dark matter. It can be confirmed with very recent observations pertaining to NGC1052-DF2 and NGC1052-DF4 galaxies. Recommended visible mass of NGC1052-DF2 $[28,29,30]$ is $\approx 1 \times 10^{8} M_{\text {Sun }}$ and its estimated dark mass is $\approx\left(\sqrt[3]{2 \times 10^{38}} / \sqrt{3.5 \times 10^{38}}\right) \approx 1.5 \times 10^{38} \mathrm{~kg}$. Sum of dark mass and visible mass is $\approx 0.35 \times 10^{9} M_{\text {Sun }}$. Corresponding flat rotation speed is $17.9 \mathrm{~km} / \mathrm{sec}$ and effective radius is $2.36 \mathrm{kpc}[34,35,36,37]$. It needs further study with respect to NGC1052-DF2 refined data.

By means of tidal mass loss [38], if dark matter shifts from satellite galaxy to its mother galaxy, based on our proposed concepts, mother galaxy's flat rotation speed must increase significantly due to increase in total matter. It is for future observational testing.

\section{Conclusion}

It is better to understand and develop models of cosmology based on well supported physical concepts rather than extraordinary physical hypothesis that demand super-normal efforts. Even though, estimated theoretical cosmic radius is 146.3 times higher the Hubble radius, estimated angular velocity is 146.3 times less than the Hubble parameter and is close to field experts advocated value. It is directly helping in estimating galactic flat rotation speeds, working radii and galactic separation distances. Further study and advanced telescopes may help in thoroughly exploring the deep space cosmic and galactic structures in a broad view.

With further study, it seems possible to understand the factors helping photon or graviton to move at 'light speed'. It is also possible to understand the factors helping universe to rotate/expand at 'light speed' and thereby, issues connected with 'hypothetical dark energy' can be resolved. Proceeding further, independent of the galactic redshift data and its controversial analysis and by continuously measuring the rate of decrease in current cosmic temperature, future cosmic expansion rate can be understood confidently.

\section{Acknowledgements}

Author Seshavatharam is grateful to Dr. E.T. Tatum for guiding with his valuable scientific thoughts on 'light speed expansion' and 'Flat Space Cosmology'. Author Seshavatharam is indebted to Professors Shri M. Nagaphani Sarma, Chairman, Shri K.V. Krishna Murthy, founder Chairman, Institute of Scientific Research in Vedas (I-SERVE), Hyderabad, India and Shri K.V.R.S. Murthy, former scientist IICT (CSIR), Govt. of India, Director, Research and Development, I-SERVE, for their valuable guidance and great support in developing this subject.

\section{COMPETING INTERESTS}

Authors have declared that no competing interests exist.

\section{References}

[1] Shamir L. Patterns of galaxy spin directions in SDSS and Pan-STARRS show parity violation and multipoles. Astrophys Space Sci. 2020; 365,136.

[2] Abhas Mitra. Why the Big Bang Model does not allow inflationary and cyclic cosmologies though mathematically one can obtain any model with favorable assumptions. New Astronomy 2014; 30: 46-50.

[3] Roger Penrose. Difficulties with Inflationary Cosmology. Annals of the New York Academy of Sciences 1989 571: 249-264.

[4] Steinhardt PJ, Abraham Loeb. Inflationary schism. Physics Letters B 2014; 736:142.

[5] Seshavatharam UVS and Lakshminarayana S. Light speed expansion and rotation of a primordial cosmic black hole universe having internal acceleration. International Astronomy and Astrophysics Research Journal 2020; 2(2): 9-27. 
[6] Seshavatharam UVS and Lakshminarayana S. To Correlate Galactic Dark and Visible Masses and to Fit Flat Rotation Speeds Via MOND Approach and Cosmic Angular Acceleration. International Astronomy and Astrophysics Research Journal 2020; 2(3): 28-43.

[7] Seshavatharam UVS and Lakshminarayana S. Light Speed Expansion and Rotation of A Very Dark Machian Universe Having Internal Acceleration. International Journal of Astronomy and Astrophysics 2020; 10:247-283.

[8] Barbour J.B. Einstein and Mach's Principle. In: Janssen M., Norton J.D., Renn J., Sauer T., Stachel J. (eds) The Genesis of General Relativity. Boston Studies in the Philosophy of Science, 2007; 250. Springer, Dordrecht. https://doi.org/10.1007/978-1-4020-4000-9_32

[9] Brans C and Dicke R H. Mach's principle and a relativistic principle of gravitation. Phys 1961 Rev. 124, 925.

[10] Arbab I Arbab. Quantization of Gravitational System and its Cosmological Consequences. Gen.Rel.Grav. 2004; 36:2465-2479.

[11] Seshavatharam UVS, Tatum E.T and Lakshminarayana S. On the role of gravitational self energy density in spherical flat space quantum cosmology. Journal of Applied Physical Science International 2015; 4(4): 228236.

[12] Seshavatharam UVS and Lakshminarayana S. Toy model of Evolving Spherical Cosmology with Flatness, Angular Velocity, Temperature and Redshift. Frontiers of Astronomy, Astrophysics and Cosmology 2015; 1(2): 90-97.

[13] Vladimir A Korotky, Eduard Masár and Yuri N Obukhov. In the Quest for Cosmic Rotation. Universe 2020; 6:14.

[14]Brownstein J R and Moffat J W Galaxy Rotation Curves Without Non-Baryonic Dark Matter. The Astrophysical Journal 2006; 636: 721-741.

[15] Milgrom M. A modification of the Newtonian dynamics as a possible alternative to the hidden mass hypothesis. Astrophysical Journal 1983; Part 1. 270:365-370.

[16] Stacy McGaugh. Predictions and Outcomes for the Dynamics of Rotating Galaxies. Galaxies 2020; 8(2): 35.

[17] Massimo Giovannini. Primordial backgrounds of relic gravitons. Prog.Part.Nucl.Phys. 2020; 103774.

[18] Christillin P. The Machian origin of linear inertial forces from our gravitationally radiating black hole Universe. Eur. Phys. J. Plus 2014; 129:175.

[19] Planck Collaboration: Planck 2015 Results. XIII. Cosmological Parameters.

[20]Eisenstein D J et al. Detection of the Baryon Acoustic Peak in the Large-Scale Correlation Function of SDSS Luminous Red Galaxies. Astrophys.J 2005; 633:560-574.

[21] César Hernández-Aguayo et al. Measuring the BAO peak position with different galaxy selections. Mon. Not. R. Astron. Soc 2020; 494(3): 3120-3130.

[22] Gamow G. The Origin of Elements and the Separation of Galaxies. Physical Review 1948; 74(4): 505.

[23] Larry Abbott. The Mystery of the Cosmological Constant. Scietific American 1998; 106-113.

[24]Perlmutter $S$ et al. Measurements of $\Omega$ and $\Lambda$ from 42 High-Redshift Supernovae. The Astrophysical Journal 1999; 517(2): 565.

[25] Riess AG et al. Observational Evidence from Supernovae for an Accelerating Universe and a Cosmological Constant. The Astronomical journal 1998; 116(3): 1009-1038.

[26] Nielsen, J.T., Guffanti, A. and Sarkar, S. Marginal Evidence for Cosmic Acceleration from Type Ia Supernovae. Scientific Reports. 2016; 6, Article No. 35596.

[27] Eric J Lerner. Observations contradict galaxy size and surface brightness predictions that are based on the expanding universe hypothesis. Monthly Notices of the Royal Astronomical Society. 2018: 477(3):31853196.

[28] Phillip Helbig. Is there a flatness problem in classical cosmology? Monthly Notices of the Royal Astronomical Society. 2012; 421(1), 561-569.

[29] Phillip Helbig. The flatness problem and the age of the Universe. Monthly Notices of the Royal Astronomical Society. 2020; 495( 4), 3571-3575.

[30] Aluri PK and Jain P. Large Scale Anisotropy due to Pre-Inflationary Phase of Cosmic Evolution. Modern. Physical Letters A 2012; 27(4):1250014.

[31] Friedmann A. On the Curvature of Space. General Relativity and Gravitation. 1999; 31(12): 1991-2000. 
[32] Hawking S.W. A Brief History of Time. Bantam Dell Publishing Group. (1988)

[33] Laura L Watkins et al. Evidence for an Intermediate-Mass Milky Way from Gaia DR2 Halo Globular Cluster Motions. The Astrophysical Journal 2019; 873:118.

[34] Alis J Deason et al. The Edge of the Galaxy. The Edge of the Galaxy, Mon. Not. R. Astron. Soc 2020; 496(3):3929-3942.

[35] Pieter van Dokkum et al. A second galaxy missing dark matter in the NGC1052 group. The Astrophysical Journal Letters 2019; 874:L5.

[36] Lewis Geraint F et al. The globular cluster population of NGC 1052-DF2: evidence for rotation. Mon. Not. R. Astron. Soc. Lett 2020; 495(1): L1-L5.

[37] Shany Danieli et al. Still Missing Dark Matter: KCWI High-resolution Stellar Kinematics of NGC1052DF2. The Astrophysical Journal Letters 2019; 874:L12.

[38] Daneng Yang, Hai-Bo Yu and Haipeng An. Self-Interacting Dark Matter and the Origin of Ultradiffuse Galaxies NGC1052-DF2 and -DF4. Phys. Rev. Lett. 2020; 125: 111105. 